\title{
Optimized ${ }^{124}$ I PET Dosimetry Protocol for Radioiodine Therapy of Differentiated Thyroid
} Cancer

\author{
Walter Jentzen, Lutz Freudenberg, Ernst G. Eising, Wilfried Sonnenschein, Jochen Knust, and Andreas Bockisch \\ Klinik für Nuklearmedizin, Universität Duisburg-Essen, Essen, Germany
}

lodine kinetics and lesion dose per administered ${ }^{131}$ l activity (LDpA) of differentiated thyroid cancer metastases were determined using ${ }^{124} \mathrm{I}$ PET. These data were analyzed to derive an optimized dosimetry protocol. Methods: We evaluated the timeactivity-concentration curves of 37 lesions in 17 patients who had undergone thyroidectomies. LDpA determination involved 124I PET images acquired at 4, 24, 48, 72, and $96 \mathrm{~h}$ after intake of a capsule containing $20-40 \mathrm{MBq}$ of ${ }^{124}$. A combination of a linear and a monoexponential or a monoexponential function only parameterized the time-activity-concentration curves. The LDpAs, calculated using data from all 5 PET time points, served as reference. The lesions were classified into 3 groups, according to potential for cure with ${ }^{131}$ I therapy: low ( $\left.\leq 5 \mathrm{~Gy} \mathrm{GBq}^{-1} ; n=14\right)$, medium (between 5 and $10 \mathrm{~Gy} \mathrm{GBq}^{-1} ; n=9$ ), or high LDpAs $\left(>10 \mathrm{~Gy} \mathrm{GBq}^{-1} ; n=14\right)$. Using the reference approach, the differences in the empiric kinetic parameters within the LDpA groups were evaluated. The reference LDpAs were compared with those derived from only 2, 3, or 4 PET data points and from 1 adapted 2-point approach. Lin's concordance correlation coefficient $\left(\rho_{\mathrm{c}}\right)$ and the mean absolute percentage deviation in LDpAs were used to assess agreement between simplified and reference approaches. Results: The effective ${ }^{124}$ I half-life, linear activity-concentration rate $(\alpha)$, and 24-h activity concentration (CpA) (the latter 2 per administered ${ }^{124}$ activity) differed significantly among the LDpA groups $(P<0.05)$. LDpAs correlated with 24-h CpAs $(r=0.94, P<$ 0.001 ). Using the 4-, 24-, and 96-h measurements, a $\rho_{\mathrm{c}}$ value of greater than or equal to 0.90 was found, and the mean absolute percentage deviation was less than or equal to $16 \%$. Similar statistical values were obtained for the adapted approach, which was based on 24- and 96-h PET data points only. Conclusion: Lesion classification into LDpA groups was feasible using a single PET scan at $\sim 24 \mathrm{~h}$. Because of the highly variable kinetics, 1 additional measurement at $\sim 96 \mathrm{~h}$ was needed to obtain a sufficiently reliable LDpA estimate. The adapted 24-96-h approach appears to be the optimal ${ }^{124}$ I protocol and is a reliable simplification of the 5-point protocol.

Key Words: PET; radionuclide therapy; dosimetry; iodine-124; differentiated thyroid cancer

J Nucl Med 2008; 49:1017-1023

DOI: 10.2967/jnumed.107.047159

Received Sep. 10, 2007; revision accepted Feb. 1, 2008.

For correspondence or reprints contact: Walter Jentzen, Klinik für Nuklearmedizin, Universität Duisburg-Essen, Hufelandstrasse 55, D-45122 Essen, Germany.

E-mail: walter.jentzen@uni-duisburg-essen.de

COPYRIGHT @ 2008 by the Society of Nuclear Medicine, Inc.

O everal groups (1-5) have successfully used ${ }^{124}$ I PET alone and with CT to guide postsurgical treatment and, in particular, radioiodine therapy in patients with differentiated thyroid carcinoma (DTC). Preradiotherapeutic ${ }^{124}$ I PET is valuable in restaging disease; dosimetric analysis of the imaging data can identify the patients most likely to benefit from radioiodine therapy and can determine safe and effective individualized therapeutic activities of radioiodine.

Our original ${ }^{124}$ I PET dosimetry protocol entailed 5 PET measurements at 4, 24, 48, 72, and $96 \mathrm{~h}$ after ${ }^{124} \mathrm{I}$ administration (2). Other ${ }^{124}$ I PET dosimetry protocols have relied on fewer measurement points. Erdi et al. (3) used a 3-point model with the first scan at $\sim 4 \mathrm{~h}$ and the last scan at $\sim 24$ or $\sim 96 \mathrm{~h}$ after radioisotope intake. Eschmann et al. (4) relied on 4 daily scans, starting at $24 \mathrm{~h}$ after ${ }^{124} \mathrm{I}$ administration. Sgouros et al. (5) generally obtained 3 PET images, at $\sim 4$, $\sim 20$, and $\sim 44$ h after ${ }^{124}$ I administration; sometimes a fourth scan was acquired at 96-144 h. However, to our knowledge, no group has yet published the results of any systematic investigation to determine the number and timing of PET scans needed for an optimized protocol that provides sufficiently reliable data on lesional absorbed dose while minimizing the quantity and time range of scans. By reducing the logistical and time demands on patients and clinic staff, such a protocol would decrease inconvenience and health care costs and help make ${ }^{124}$ I PET dosimetry more widely available and user-friendly. We therefore conducted the present protocol optimization study.

\section{MATERIALS AND METHODS}

\section{Study Design}

We retrospectively compared our original 5-measurement-point ${ }^{124}$ I PET dosimetry protocol (the reference approach) with various alternative protocols, using data from fewer measurement times for accuracy in determining DTC lesional radiation doses. The comparison was made within each of 3 lesion groups, classified on the basis of potential for cure by radioiodine therapy as reflected by a low, medium, or high lesion dose per administered activity of ${ }^{131} \mathrm{I}$ (LDpA) in $\mathrm{Gy}_{\mathrm{GBq}}{ }^{-1}$. The first part of the study comprised a permutation analysis examining all evaluable alternative protocols. The second part of the study, the empiric analysis, used findings 
from the permutation analysis and from the reference iodine kinetic parameters to develop and assess one adapted approach, offering still greater simplicity.

\section{Patients and Lesions}

The study included 17 patients (10 women, 7 men) who had at least 1 metastatic DTC lesion. Mean $( \pm \mathrm{SD})$ age was $56 \pm 16 \mathrm{y}$ (median, $53 \mathrm{y}$; range, 32-83 y). The 17 patients had a total of 37 iodine-avid metastatic lesions (19 of which involved the lymph nodes, 5 the lungs, 10 the bone, and 3 other sites). DTC histology was papillary in 12 and follicular in 5 cases. Thirteen of the 17 patients had undergone 1-7 previous radioiodine therapies (mean \pm $\mathrm{SD}, 2.7 \pm 1.8$; median, 2$)$, with a mean ( $\pm \mathrm{SD}$ ) cumulative activity of $16.9 \pm 14.3 \mathrm{GBq}$ (median, $14 \mathrm{GBq}$; range, 3-52 GBq). The study dosimetry took place before the first or second radioiodine therapy in 4 patients and before the third to eighth radioiodine therapy in the remaining 9 patients.

\section{Tomograph, Acquisition, and Image Reconstruction}

Tomograph. PET was performed using a scanner (ECAT EXACT $\mathrm{HR}^{+}$; CTI/Siemens) at approximately 4, 24, 48, 72, and $96 \mathrm{~h}$ after oral intake of a capsule containing $20-40 \mathrm{MBq}$ of ${ }^{124}$ I-sodium iodide. Details on radionuclide production and calibrated activity measurement were published elsewhere (6). ${ }^{124}$ I was given when serum thyroid-stimulating hormone levels were greater than or equal to $25 \mu \mathrm{L}^{-1}$ after either at least $4 \mathrm{wk}$ without thyroid hormone ( $n=10)$ or $24 \mathrm{~h}$ after the second of 2 consecutive daily intramuscular injections of recombinant human thyroid-stimulating hormone (Genzyme Corp.) $(n=7)$.

Acquisition. PET measurements were acquired in 3-dimensional mode, with scanning from the thigh or abdomen to the head using 5-8 bed positions with an emission time of $281 \mathrm{~s}$ each. The data were corrected for attenuation by a measured transmission scan (138 s/bed position) and standard scatter correction.

Image Reconstruction. Image reconstruction was performed after Fourier rebinning with attenuation-weighted ordered-subset expectation maximization using 8 subsets, 2 iterations, and a 5 -mm gaussian filter. The resulting PET image had $256 \times 256$ voxels (voxel size, $1.72 \times 1.72 \times 2.43 \mathrm{~mm}^{3}$ ). In clinical applications, the image resolution expressed as full width at half maximum (FWHM) was $8 \mathrm{~mm}(6)$.

\section{Determination of the Activity Concentration}

The measured activity concentration was obtained using circular FWHM-sized regions of interest (ROIs) (8-mm diameter) (6). This resolution ROI technique has been proposed for determining recovery coefficients in phantom measurements needed for quantification and, therefore, should also be applied to patient data (7). For large lesions (diameter $\geq 1.5 \mathrm{~cm}$ ), the mean activity concentration was determined by averaging the concentrations in FWHMsized ROIs drawn in the centers of the slice with the maximum lesion activity concentration and of 2 corresponding adjacent slices $( \pm 1)$. The concentrations of smaller lesions (diameter $<1.5 \mathrm{~cm}$ ) were obtained using a line profile (the mean activity concentration in the FWHM-sized ROI vs. axial distance), which was fitted with a gaussian function; the corresponding gaussian amplitude was taken as the lesion activity concentration. Images were analyzed with software (ECAT 7.2.1; CTI/Siemens).

\section{Lesion Volume and Recovery Correction}

Lesion Volume. Lesion volumes were obtained using the PETbased iterative thresholding method; the lesions were assumed to be ellipsoidal (8). An effective lesion diameter was calculated to apply the recovery correction method; that is, an effective sphere of the same volume can replace the ellipsoidal lesion volume. The mean absolute percentage deviation between CT and PET was $\sim 10 \%$ for lesion volumes from 0.8 to $7.5 \mathrm{~mL}$ and $\sim 15 \%$ for volumes greater than $7.5 \mathrm{~mL}(8)$.

Recovery Correction. For lesions of simple geometric shape, recovery effects can be corrected for by using recovery coefficients $(R C s)$ determined in phantom measurements, as described elsewhere (6) for the study PET system. The measured activity concentration, $C_{\mathrm{msd}}$, can be corrected for by using the equation $C_{\text {cor }}=C_{\text {msd }} R C^{-1}$ (9). Remarkably, even for large lesions (e.g., 37-mm diameter), the ${ }^{124}$ I recovery coefficient determined for our PET system was 0.78 , not unity, a phenomenon mainly associated with the complex decay scheme of the radionuclide (6). The reliability of the recovery correction depended on the lesion volume determination. Phantom measurements mimicking in vivo conditions in patients with DTC demonstrate that the maximum absolute percentage deviation between expected and measured ${ }^{124} \mathrm{I}$ activity concentrations after applying recovery correction was about $10 \%$ for sphere volumes greater than or equal to $1 \mathrm{~mL}$ and about $20 \%$ for sphere volumes of $0.5 \mathrm{~mL}(6)$.

\section{Parameterizing Lesional Time-Activity-Concentration Curve and Estimating Lesional Cumulated Activity Concentration (CAC)}

Parameterizing the Time-Activity-Concentration Curve. The lesional time-activity-concentration curve was parameterized to determine the CAC using 1 of 2 functional models (Fig. 1):

$$
\begin{aligned}
& C_{1}(t)=C_{0} \cdot e^{-\ln 2 \cdot \frac{t}{{ }^{124} I_{\mathrm{eff}}}} \\
& C_{2}(t)= \begin{cases}\alpha \cdot t & t \leq t_{s} \\
C_{0} \cdot e^{-\ln 2 \cdot \frac{t}{124} I_{\mathrm{eff}}} & t>t_{s}\end{cases} \\
& \text { Eq. } 1 \\
& \text { Eq. } 2
\end{aligned}
$$

where $C_{0}$ was the (extrapolated) concentration at time zero, ${ }^{124} \mathrm{I}_{T_{\text {eff }}}$ the effective half-life of ${ }^{124} \mathrm{I}$, and $\alpha$ the initial linear time-activityconcentration rate. The time, $t_{s}$, indicated the point of intersection of the linear function $(\alpha \cdot t)$ with the monoexponential function

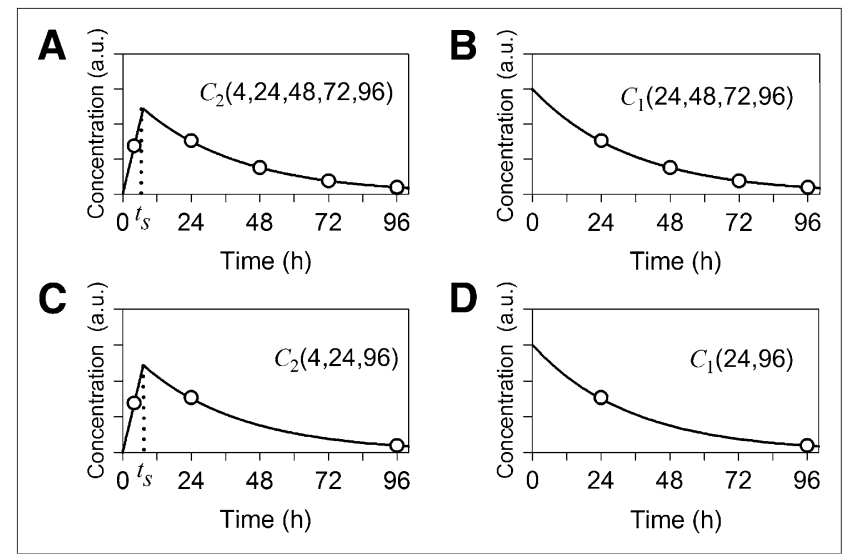

FIGURE 1. Schematic representation of time-activityconcentration curves of reference approach (A) and representative alternative approaches (B-D) to estimate LDpA. a.u. = arbitrary units. 
(Fig. 1A). The corresponding CAC was the integral of $C(t)$ from zero to infinity. To avoid ambiguity caused by the limited number of data points, no curve-fitting procedure was used. The parameters of the functions $C_{1}(t)$ and $C_{2}(t)$ were determined algebraically: $C_{1}(t)$ required at least 2 points, whereas $C_{2}(t)$ required 3 points including the 4-h measurement (for the slope $\alpha$ ) plus 2 later points $\left(C_{0},{ }^{124} \mathrm{I}_{T_{\mathrm{eff}}}\right)$. A linear regression analysis (plot of logarithm of activity concentration vs. time) was undertaken to obtain the 2 parameters, $C_{0}$ and ${ }^{124} \mathrm{I}_{T_{\text {eff }}}$, in the exponential function.

Estimating the CAC. The functional model $C_{1}$ was chosen to estimate the CAC unless the 4-h PET data were included in the dataset, in which case the functional model $C_{2}$ was used. Two-point models including the 4-h PET measurement were excluded from the analysis because of their inability to represent accurately the timeactivity-concentration curve. In total, 21 alternatives to the reference approach were analyzed: six 2-point, ten 3-point, and five 4-point approaches; representative examples appear in Figures 1B, $1 \mathrm{C}$, and 1D. The following name convention was chosen: the first symbol, $C_{1}$ or $C_{2}$, denoted the functional model describing the timeactivity-concentration curve and the numbers in parentheses were the PET measurement time points in hours after ${ }^{124}$ I administration. For instance, $C_{1}(24,96)$ (Fig. 1D) is a 2-point model using 24- and 96-h PET measurements, and the time-activity-concentration curve is described by a monoexponential function. The reference approach was termed $C_{2}(4,24,48,72,96)$ (Fig. 1A).

\section{Group Classification and LDpA}

Group Classification. The studied lesions were classified into 3 groups, according to the likelihood of success of radioiodine therapy, based on the projected LDpA determined using the reference approach: low $\left(\leq 5 \mathrm{~Gy} \mathrm{GBq}^{-1}\right)$, medium $\left(>5 \mathrm{~Gy} \mathrm{GBq}^{-1}\right.$ to $\leq 10 \mathrm{~Gy} \mathrm{GBq}^{-1}$ ), and high $\mathrm{LDpA}\left(>10 \mathrm{~Gy} \mathrm{GBq}^{-1}\right)$. These cutoffs were calculated on the basis of the findings of Maxon et al. (10) that minimum lesion radiation doses of 85-140 Gy are necessary to successfully treat nodal metastases and on the basis of the maximum single activity of $15 \mathrm{GBq}$ found by our department.

The LDpA was calculated as follows:

$$
\mathrm{LDpA}=\Delta \cdot \frac{\int_{0}^{\infty} C(t) d t}{A_{t r} \cdot \rho},
$$

where $C(t)$ was the ${ }^{131} \mathrm{I}$ activity concentration corrected for the difference in physical half-lives of ${ }^{124} \mathrm{I}$ (tracer nuclide) and ${ }^{131} \mathrm{I}$ (therapeutic nuclide), $\Delta$ the equilibrium ${ }^{131} \mathrm{I}$ dose constant for nonpenetrating radiation $\left(0.11 \mathrm{~Gy} \mathrm{~g} / \mathrm{MBq}^{-1} \mathrm{~h}^{-1}\right)(11), A_{t r}$ the tracer activity shortly after capsule administration, and $\rho$ the tissue density $\left(1 \mathrm{~g} / \mathrm{mL}^{-1}\right) .{ }^{124} \mathrm{I}$ and ${ }^{131} \mathrm{I}$ kinetics were assumed to be identical; therefore, the CAC could be transformed if the ${ }^{124} \mathrm{I}$ lesion kinetics were known. The self-irradiation (lesion-to-lesion) absorbed dose was calculated using the MIRD formula.

\section{Statistics}

Comparisons of alternatives to the reference approach were performed separately for each LDpA group using Lin's concordance correlation coefficients $\left(\rho_{\mathrm{c}}\right)$ along with $95 \%$ confidence intervals (CIs) (12) and the mean absolute percentage deviation $(\Delta)$ between the alternatives and the reference approach. The $\rho_{\mathrm{c}}$ value evaluates the degree to which paired measures fall on the $45^{\circ}$ line through the origin. The compared approaches are regarded as equivalent if the $\rho_{\mathrm{c}}$ is above a specified threshold (agreement increases as $\rho_{\mathrm{c}}$ approaches 1.0). Whether agreement is excellent, moderate, or poor is a subjective decision. Partik's criteria (13) grade $\rho_{\mathrm{c}}$ values more than 0.95 as excellent and values more than 0.90 as very good. In the present study, approaches with $\rho_{\mathrm{c}}$ values of at least 0.90 were regarded as equivalent.

Differences within the LDpA groups were evaluated by the Kruskal-Wallis ANOVA on ranks, a nonparametric test, and the pairwise differences (between-group analysis) were evaluated by the Dunn test. Statistically significant differences were assumed at a $P$ value of less than 0.05 . Software (Stata 9.1; StataCorp) was used for computation.

\section{RESULTS}

\section{Reference lodine Kinetics and Dosimetry Data}

Table 1 summarizes selected key reference kinetic variables, and Figure 2 shows examples of the reference timeactivity-concentration curve and fits for each reference LDpA group. The LDpA ranged from 1.1 to $215 \mathrm{~Gy}$

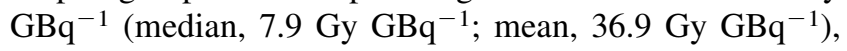
and high intra- and interpatient variability was observed. The $24-\mathrm{h}{ }^{124} \mathrm{I}$ activity concentration ranged from 2.3 to 367 $\mathrm{kBq} / \mathrm{mL}^{-1}$ (median, $22 \mathrm{kBq} / \mathrm{mL}^{-1}$; mean, $77 \mathrm{kBq} / \mathrm{mL}^{-1}$ ), and the $24-\mathrm{h}{ }^{124} \mathrm{I}$ lesional uptake ranged from $0.01 \%$ to $8.4 \%$ (median, $0.11 \%$; mean, $0.56 \%$ ). The lesion volume ranged from 0.7 to $28 \mathrm{~mL}$ (median, $1.0 \mathrm{~mL}$; mean, $2.4 \mathrm{~mL}$ ). The effective half-life, the $\alpha$ per administered ${ }^{124}$ I activity (RpA), and the 24-h activity concentration per administered ${ }^{124}$ I activity (CpA) showed significant differences among the LDpA groups $(P<0.002$, ANOVA). Specifically, the effective half-lives, 24-h $\mathrm{CpA}$, and RpA deviated significantly between the low- and high-LDpA groups $(P<0.05$, Dunn test) as did the 24-h CpA and RpA between the medium- and high-LDpA groups $(P<0.05$, Dunn test $)$. The interception points, $t_{S}$, showed no significant differences among the reference LDpA groups. The median (mean $\pm \mathrm{SD}$ ) interception point for all 37 lesions was $8.0 \mathrm{~h}$ $(8.8 \pm 4.2 \mathrm{~h})$.

TABLE 1

Reference Kinetic Parameters in DTC Metastases by LDpA Group

\begin{tabular}{lccrrrr}
\hline LDpA group & ${ }^{131} \mathrm{I} \operatorname{LDpA}\left(\frac{\mathrm{Gy}}{\mathrm{GBq}}\right)$ & ${ }^{131} \mathrm{I}_{T_{\text {eff }}}(\mathrm{h})$ & ${ }^{131} \mathrm{I}_{\mathrm{s}_{\mathrm{S}}}(\mathrm{h})$ & ${ }^{124} \mathrm{I}_{T_{\text {eff }}}(\mathrm{h})$ & ${ }^{124} \mathrm{I} \mathrm{RpA}\left(\frac{\mathrm{kBq}}{\mathrm{mL} \cdot \mathrm{h} \cdot \mathrm{MBq}}\right)$ & ${ }^{24-\mathrm{h}}{ }^{124} \mathrm{I} \mathrm{CpA}\left(\frac{\mathrm{kBq}}{\mathrm{mL} \cdot \mathrm{MBq}}\right)$ \\
\hline Low & $2.2(2.6)$ & $17(26)$ & $5.5(6.8)$ & $16(23)$ & $0.09(0.14)$ & $0.25(0.28)$ \\
Medium & $7.9(7.9)$ & $57(51)$ & $10.0(9.9)$ & $45(41)$ & $0.09(0.12)$ & $0.70(0.76)$ \\
High & $92.1(89.7)$ & $61(73)$ & $8.0(9.6)$ & $47(54)$ & $0.73(0.85)$ & $5.74(6.03)$
\end{tabular}

Data are median values, with mean values in parentheses. 


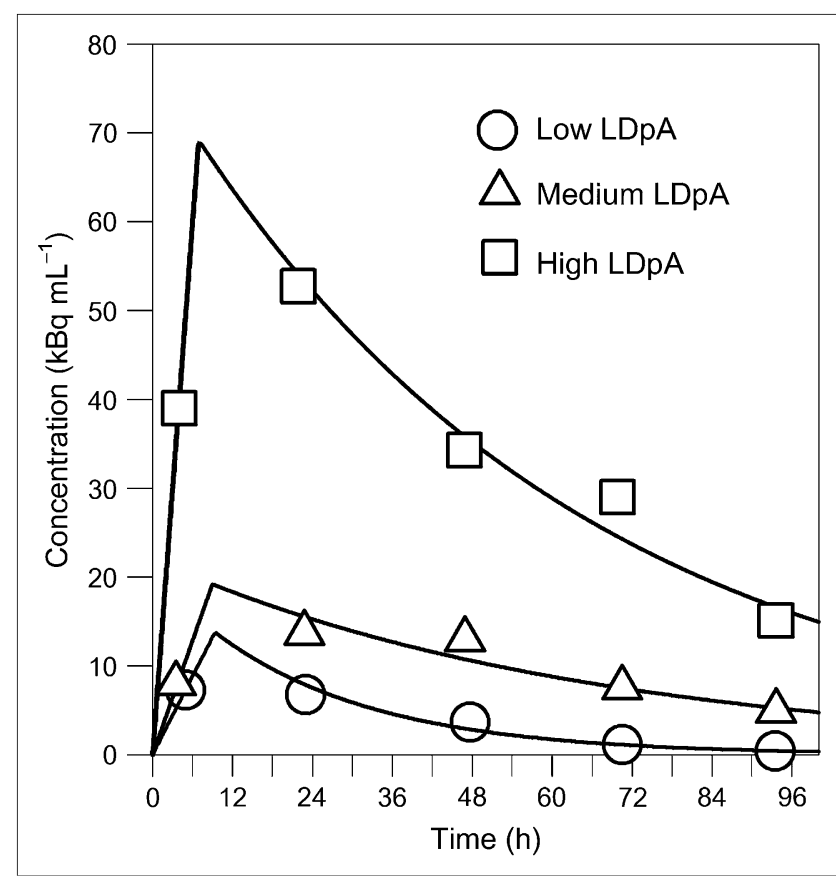

FIGURE 2. Representative examples of ${ }^{124} \mid$ time-activityconcentration curves of low-, medium-, and high-LDpA groups. Curves were determined using reference approach $C_{2}(4,24,48,72,96)$. Open circles are data taken from serial PET images.

The reference 24-h CpAs correlated well with the reference LDpAs (Pearson correlation coefficient, 0.94; $P<$ 0.001 ) and therefore appeared to be a good alternative to the reference LDpA for the group classification. If the 24-h CpA values only were used, the minimum number of lesions ( $n=$ 3 ; lesions 12,17, and 22) was miscategorized relative to the reference LDpA when the following reference CpA thresholds were selected: low group $(n=14)$, less than $0.45 \mathrm{kBq} /$ $\mathrm{mL}^{-1} \mathrm{MBq}^{-1}$; medium group $(n=8), 0.45-1.0 \mathrm{kBq} / \mathrm{mL}^{-1}$ $\mathrm{MBq}^{-1}$; high group $(n=15)$, greater than $1.0 \mathrm{kBq} / \mathrm{mL}^{-1}$ $\mathrm{MBq}^{-1}$.

\section{Permutation Analysis}

Analysis of Lin's correlation coefficients for all studied alternative approaches showed that only $2, C_{1}(24,96)$ and
$C_{2}(4,24,96)$, were of interest for protocol optimization that substantially reduced the number of PET scans. Table 2 lists Lin's coefficients and the mean absolute percentage deviations in LDpA for some representative approaches. Figure 3 shows Lin's concordance correlation plots. Figure 4 depicts the individual absolute percentage deviation in LDpA of each lesion between $C_{1}(24,96)$ (panel A) or $C_{2}(4,24,96)$ (panel B) versus the reference approach. Using $C_{1}(24,96)$, the mean absolute percentage deviation was $8 \%$ (maximum, 26\%) for the medium-LDpA group and 5\% (maximum, 11\%) for the high-LDpA group, whereas the mean absolute percentage deviation of $29 \%$, a maximum percentage deviation of $90 \%$, and a $\rho_{\mathrm{c}}$ value of less than 0.90 for the low-LDpA group was unacceptable. Adding the 4-h data point, specifically using $C_{2}(4,24,96)$, the mean absolute percentage deviation for the low-LDpA group decreased to $16 \%$ (maximum, 34\%) and the $\rho_{\mathrm{c}}$ value increased to 0.92 . The medium-LDpA group showed an acceptable mean absolute percentage LDpA deviation for the $C_{1}(24,96)$ approach; however, if the 4-h data point was not considered, the $\rho_{\mathrm{c}}$ value was slightly less than 0.90 . This finding was probably associated with the small number of lesions $(n=9)$ in this group. Thus, including the 4-h data point appeared to be necessary for protocol optimization, especially in the low-LDpA group, but of minor relevance to protocol optimization in the medium- and, particularly, the high-LDpA groups.

\section{Empiric Analysis}

The permutation analysis showed that the $C_{1}(24,96)$ approach produced acceptable estimates of the absorbed dose for lesions that were in the high- or medium- but not in the low-LDpA group. This is because the LDpA fraction arising from the integral of $C(t)$ from zero to $t_{s}$, that is, the linear contribution to the total LDpA, was often larger for lesions in the low- than for lesions in the medium- or highLDpA groups. To compensate for this phenomenon, a fixed interception point was chosen to estimate the linear contribution, as no significant intergroup difference was found for the interception points. Specifically, the adapted approach used data points from only 2 (24- and 96-h) PET scans and an assumed fixed interception point of $8 \mathrm{~h}$ (the median for

TABLE 2

Lin's Concordance Correlation Coefficients and $95 \% \mathrm{Cl}$ and Mean Absolute Percentage Deviations in LDpA $(\Delta$ in \%) Between Alternative and Reference Approaches

\begin{tabular}{|c|c|c|c|c|c|c|c|c|c|}
\hline \multirow[b]{2}{*}{ Approach } & \multicolumn{3}{|c|}{ Low LDpA $(n=14)$} & \multicolumn{3}{|c|}{ Medium LDpA $(n=9)$} & \multicolumn{3}{|c|}{ High LDpA $(n=14)$} \\
\hline & $\rho$ & $95 \% \mathrm{Cl}$ & $\Delta$ & $\rho$ & $95 \% \mathrm{Cl}$ & $\Delta$ & $\rho$ & $95 \% \mathrm{Cl}$ & $\bar{\Delta}$ \\
\hline$C_{1}(24,96)$ & 0.82 & $0.65-0.99$ & 29 & 0.89 & $0.76-1.02$ & 8 & 0.99 & $0.98-1.00$ & 5 \\
\hline$C_{1}(48,96)$ & 0.31 & $0.06-0.57$ & 62 & 0.84 & $0.64-1.05$ & 12 & 0.99 & $0.99-1.00$ & 6 \\
\hline$C_{2}(4,24,96)$ & 0.92 & $0.84-1.00$ & 16 & 0.91 & $0.79-1.03$ & 8 & 0.99 & $0.97-1.00$ & 6 \\
\hline$C_{2}(4,48,96)$ & 0.74 & $0.55-0.93$ & 32 & 0.95 & $0.88-1.02$ & 5 & 0.99 & $0.99-1.00$ & 5 \\
\hline$C_{2}(4,24,48,96)$ & 0.99 & $0.98-1.00$ & 5 & 0.97 & $0.93-1.01$ & 4 & 0.99 & $0.97-1.00$ & 5 \\
\hline Adapted $C_{2}(24,96)$ & 0.92 & $0.84-1.00$ & 12 & 0.91 & $0.78-1.03$ & 8 & 0.99 & $0.98-1.00$ & 5 \\
\hline
\end{tabular}




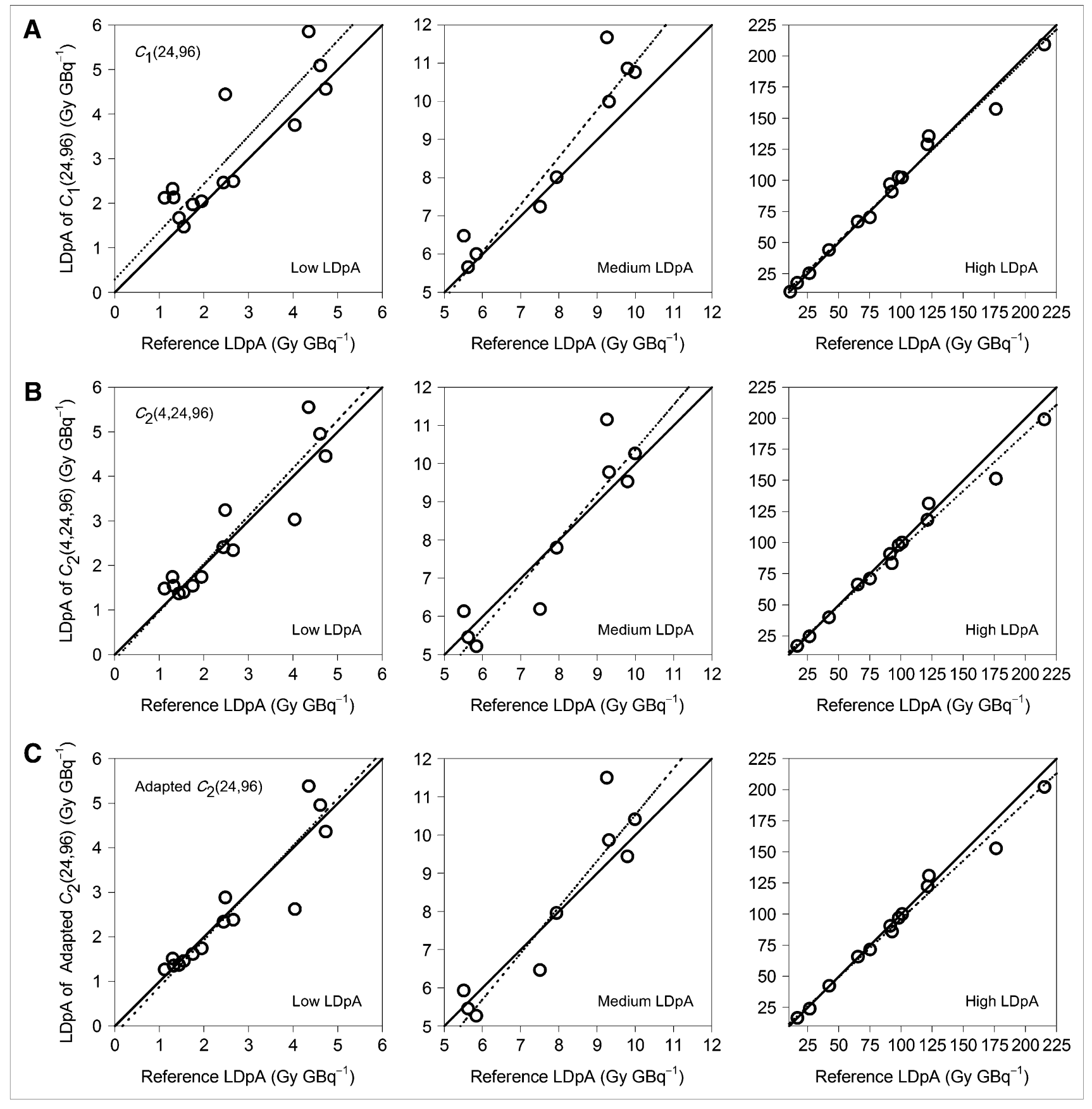

FIGURE 3. Lin's concordance correlation plots for $C_{1}(24,96)(\mathrm{A}), C_{2}(4,24,96)(\mathrm{B})$, and adapted $C_{2}(24,96)$ approaches $(\mathrm{C})$, along with line of identity (solid) and reduced major axis regression line (dotted).

all 37 lesions). The absorbed dose of this adapted 24-96-h approach was estimated using the functional model $C_{2}$ :

$$
\begin{aligned}
\mathrm{LDpA}= & \frac{\Delta}{\rho} \cdot{ }^{124} \mathrm{I}_{\mathrm{CpA}_{24}} \cdot e^{\ln 2 \cdot\left(\frac{t_{24}}{{ }^{124} \mathrm{I}_{\mathrm{Teff}}}-\frac{{ }^{131_{\mathrm{I}}}{ }_{t_{S}}}{{ }^{131_{\mathrm{I}}}{ }_{T_{\mathrm{eff}}}}\right)} \\
& \cdot\left(0.5 \cdot{ }^{131} \mathrm{I}_{t_{S}}+\frac{131_{\mathrm{I}_{\mathrm{Teff}}}}{\ln 2}\right)
\end{aligned}
$$

where ${ }^{124} \mathrm{I}_{\mathrm{CpA}_{24}}$ is the $24-\mathrm{h} \mathrm{CpA}$ for ${ }^{124} \mathrm{I}$ and ${ }^{124} \mathrm{I}_{T_{\text {eff }}}$ is the effective half-life calculated from 24- and 96-h ${ }^{124}$ I PET measurements via linear regression analysis. ${ }^{131} \mathrm{I}_{T_{\text {eff }}}$ is the effective half-life for ${ }^{131} \mathrm{I}$. The time of PET measurement at $\sim 24 \mathrm{~h}$ is designated by the symbol $t_{24}$ and ${ }^{131} \mathrm{I}_{t_{\mathrm{s}}}\left(\approx{ }^{124} \mathrm{I}_{t_{\mathrm{s}}}\right)$ is the fixed interception point of $8 \mathrm{~h}$. The other symbols are defined earlier in the description of Equation 3. Table 2 lists the relevant statistical variables, and Figure 3C shows Lin's concordance correlation plots for this adapted approach. Lin's coefficients were identical with those of $C_{2}(4,24,96)$. Figure $4 \mathrm{C}$ depicts the individual absolute percentage deviation of each lesion with the adapted $C_{2}(24,96)$ versus the reference approach. The mean absolute percentage devia- 


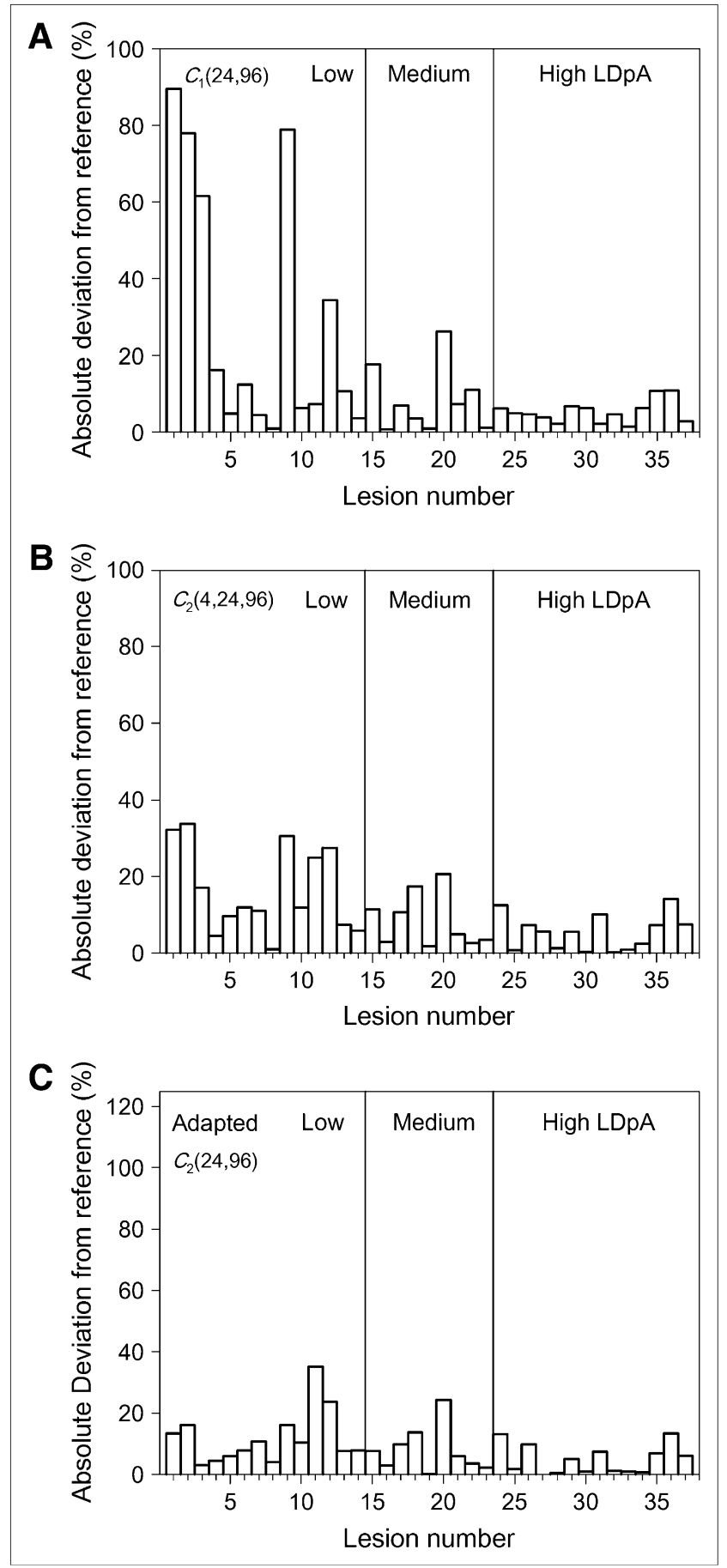

FIGURE 4. Absolute percentage deviations in LDpA for each of 37 studied lesions between $C_{1}(24,96)(A), C_{2}(4,24,96)(B)$, or adapted $C_{2}(24,96)(C)$ versus reference approach.

tion was $12 \%$ (maximum, 35\%) for the low-, $8 \%$ (maximum, $14 \%$ ) for the medium-, and 5\% (maximum, 13\%) for the high-LDpA group.

\section{DISCUSSION}

Using the reference approach (5 PET measurement points from 4 to $96 \mathrm{~h}$ ), the present study found broadly similar radioiodine kinetics to those observed by other groups investigating ${ }^{124}$ I PET dosimetry, albeit we noted wider ranges and higher maximum values than did those groups (3-5). Our LDpA range was 1.1-215 Gy GBq ${ }^{-1}$. Eschmann et al. (4), who used a combination of the Marinelli formula and MIRDOSE3.1 to estimate the absorbed dose, obtained an LDpA range of $10-21 \mathrm{~Gy} \mathrm{GBq}^{-1}$ in 4 metastatic lesions in 2 patients. A mean dose range of 0.4-24.4 Gy GBq ${ }^{-1}$ (3 lesions) was reported by Erdi et al. (3) and a mean dose range of $0.08-49 \mathrm{~Gy} \mathrm{GBq}^{-1}$ (56 lesions) by Sgouros et al. (5), who performed a point-kernel-based calculation and 3dimensional internal dosimetry. The ${ }^{124}$ I effective half-life in metastases ranged from 6 to $80 \mathrm{~h}$ in the present study, 14 to $43 \mathrm{~h}$ in the study of Erdi et al. (3), and 22 to $65 \mathrm{~h}$ in the study of Eschmann et al. (4). No effective ${ }^{124}$ I half-lives were reported by Sgouros et al. (5). The present work also confirmed and extended the observation of the study of Sgouros et al. (5) (the only other published investigation of more than a handful of lesions) that in DTC metastases, LDpAs and ${ }^{124}$ I effective half-lives showed a broad variability, even within a single patient; for example, an individual could have both low- and medium-/high-LDpA lesions.

The clinical course of DTC is often favorable, and standardized radioiodine therapy is well established and frequently successful. In selected patients, an individual therapy needs to be performed at the limits, and prospective dosimetry is required. A reliable ${ }^{124} \mathrm{I}$ dosimetry protocol necessitates multiple PET measurements over a few days. This entails substantial clinical resources and costs. The 5-d protocol (4-96 h) is certainly the most accurate among the approaches investigated. More PET data points are even more accurate, but a cost-benefit protocol implies a tradeoff between accuracy and effort that should relate to the importance of the clinical decision.

The LDpAs determined in this work were subject to uncertainties. The physical errors mainly arose from the determination of the lesion volume and of the ${ }^{124}$ I activity concentrations including recovery correction. The volume was determined assuming that the lesions were ellipsoids, as more complicated shapes cannot be easily handled in a clinical setting. The error range of the volume determination was $\sim 10 \%$ to $\sim 15 \%(8)$ and the size-dependent recovery coefficients were influenced by the accuracy of the volume determination (6). Phantom measurements mimicking in vivo conditions showed that the maximum inaccuracy of the ${ }^{124} \mathrm{I}$ activity concentration after recovery correction was $\sim 10 \%$ for spheres with an inner volume of $1 \mathrm{~mL}$ or more and $\sim 20 \%$ for spheres with a $0.5-\mathrm{mL}$ volume (6). Other possible uncertainties are biologic factors occurring during ${ }^{124} \mathrm{I}$ diagnostic procedures and ${ }^{131} \mathrm{I}$ therapy. The estimated absorbed dose based on pretreatment diagnostics and the actual dose determined after therapy differ because of a faster-than-expected release of radioiodine in the immediate aftermath of radioiodine therapy (10); the reason is probably the immediate onset of therapeutic effects during ${ }^{131} \mathrm{I}$ treatment. Finally, radioiodine distribution is known to be inhomogeneous. The inaccuracies of biologic factors are a priori unquantifiable. 
In total, the estimated physics-related error range using the reference approach may total $20-30 \%$. This error range is acceptable for an optimized ${ }^{124} \mathrm{I}$ dosimetry protocol to provide pretherapeutic lesional absorbed dose estimates in patients scheduled for high-dose ${ }^{131} \mathrm{I}$ therapy when 2 or more PET scans can be avoided.

Physicians are primarily interested in whether the administration of reasonable ${ }^{131} \mathrm{I}$ activities is likely to achieve a clinically meaningful response or likely to fail. In association with the radiation dose threshold for successful treatment (5-10 Gy $\mathrm{GBq}^{-1}$ or larger), the $\mathrm{LDpA}$ is the key to providing this information. Our permutation analysis suggested that the 2 earliest ( 4 and $24 \mathrm{~h}$ ) and the latest PET data points $(96 \mathrm{~h}$ ) provided robust estimates of the LDpA; that is, the $C_{2}(4,24,96)$ approach using these 3 data points yielded a $\rho_{\mathrm{c}}$ greater than or equal to 0.90 and a mean absolute percentage deviation less than or equal to $16 \%$ relative to the reference approach. More important, similar Lin's coefficients and absolute deviations were found for the adapted 24-96-h approach, which uses only 2 PET scans and a fixed interception point of $8 \mathrm{~h}$. Thus, this 2-point protocol appears to offer the best trade-off between accuracy and effort. Especially if the clinical workload is heavy,

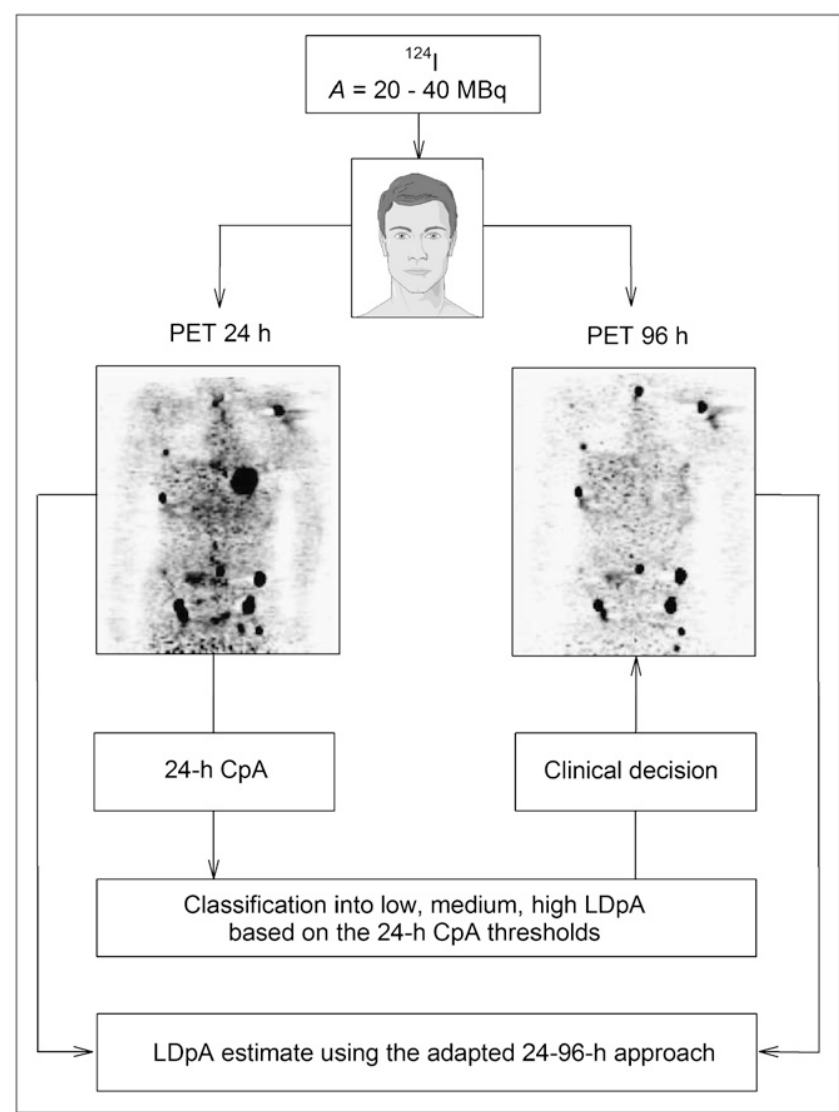

FIGURE 5. Flow chart illustrating optimized dosimetry protocol using adapted 24-96-h approach for patients with DTC undergoing ${ }^{124}$ I PET before planned radioiodine therapy. Clinical decision to perform 96-h measurement depends on LDpA classification; that is, low $24-\mathrm{h} \mathrm{CpA}$ indicates that radioiodine therapy is likely to fail. the adapted 24-96-h approach appears to be a good alternative, as the only potential cure is either radioiodine therapy or surgery. A flow chart illustrating the optimized dosimetry protocol (i.e., the adapted 24-96-h approach) is given in Figure 5. The clinical decision to perform the 96-h measurement depends on the LDpA classification; that is, a very low 24-h CpA (low-LDpA classification) implies that radioiodine therapy is likely to fail and surgery may be more appropriate.

\section{CONCLUSION}

Radioiodine kinetics and, hence, LDpA of DTC metastases showed great variability. For that reason, multiple ${ }^{124} \mathrm{I}$ PET investigations are required. The 5-point protocol is certainly the most accurate among the approaches investigated, but the adapted 24-96-h approach using 2 points is an optimal dosimetry protocol when the clinical workload is particularly heavy, reducing logistical and time demands for patients and caregivers and clinical costs.

\section{ACKNOWLEDGMENTS}

We are indebted to Robert J. Marlowe for critically reviewing the manuscript and for providing substantive scientific critique. We thank Dr. Annette Böckenhoff for her suggestions and guidance in the statistical analysis.

\section{REFERENCES}

1. Freudenberg LS, Antoch G, Jentzen W, et al. Value of ${ }^{124}$ I-PET/CT in staging of patients with differentiated thyroid cancer. Eur Radiol. 2004;14:2092-2098.

2. Freudenberg LS, Jentzen W, Görges R, et al. ${ }^{124}$ I-PET dosimetry in advanced differentiated thyroid cancer: therapeutic impact. Nuklearmedizin. 2007;46:121-128.

3. Erdi YE, Macapinlac H, Larson SM, et al. Radiation dose assessment for I-131 therapy of thyroid cancer using I-124 PET imaging. Clin Pos Imag. 1999;2:41-46.

4. Eschmann SM, Reischl G, Bilger K, et al. Evaluation of dosimetry of radioiodine therapy in benign and malignant thyroid disorders by means of iodine-124 and PET. Eur J Nucl Med Mol Imaging. 2002;29:760-767.

5. Sgouros G, Kolbert KS, Sheikh A, et al. Patient-specific dosimetry for I-131 thyroid cancer therapy using I-124 PET and 3-dimensional-internal dosimetry (3D-ID) software. J Nucl Med. 2004;45:1366-1372.

6. Jentzen W, Weise R, Kupferschläger J, et al. Iodine-124 PET dosimetry in differentiated thyroid cancer: recovery coefficient in 2D and 3D modes for PET(/CT) systems. Eur J Nucl Med Mol Imaging. 2007;35:1619-7070.

7. International Eletrotechnical Commission. International Standard IEC 61675-1: Radionuclide Imaging Devices-Characteristics and Test Conditions-Part 1: Positron Emission Tomographs. Geneva, Switzerland: International Eletrotechnical Commission; 1998.

8. Jentzen W, Freudenberg LS, Heinze M, et al. Segmentation of PET volumes by iterative image thresholding. J Nucl Med. 2007;48:108-114.

9. Knoop BO, Geworski L, Hofmann M, et al. Use of recovery coefficients as a test of system linearity of response in positron emission tomography. Phys Med Biol. 2002; 47:1237-1254.

10. Maxon HR, Thomas SR, Samaratunga RC. Dosimetric considerations in the radioiodine treatment of macrometastases and micrometastases from differentiated thyroid cancer. Thyroid. 1997;7:183-187.

11. Snyder WS, Ford MR, Warner GG, et al. "S," Absorbed Dose per Unit Cumulated Activity for Selected Radionuclides and Organs. MIRD Pamphlet 11. Reston, VA: Society of Nuclear Medicine; 1975.

12. Lin L, Hedayat AS, Sinha B, et al. Statistical methods in assessing agreement: models, issues, and tools. J Am Stat Assoc. 2002;97:257-270.

13. Partik BL, Stadler A, Schamp S, et al. 3 D versus 2 D ultrasound: accuracy of volume measurement in human cadaver kidneys. Invest Radiol. 2002;37:489-495. 